\title{
Revisión de las Características de Modelado y Optimización para el Diseño del Sistema de Suspensión Macpherson
}

\author{
William H. Vega( ${ }^{(1,2)}$, Edilberto A. Llanes-Cedeño(1), Jaime V. Molina ${ }^{(1)}$ y Juan C. Rocha-Hoyos ${ }^{(1)}$ \\ (1) Univ. Particular Internacional SEK, Fac. de Arquitectura e Ingenierías, Casilla 17-03-02, Quito - Ecuador \\ (e-mail: wvega.mdm@uisek.edu.ec; carlos.rocha@uisek.edu.ec) \\ (2) Min. de Educación Ecuador, Dpto. de Administración Escolar Coordinación zona 1 educación, \\ Casilla 10-02-01, Ibarra - Ecuador
}

Recibido Abr. 4, 2018; Aceptado May. 30, 2018; Versión final Jun. 23, 2018, Publicado Dic. 2018

\begin{abstract}
Resumen
El objetivo de este trabajo fue caracterizar los procesos de diseño en los contextos de aplicación de software de simulación, resaltando las técnicas predictivas de multicuerpos, elementos finitos y modelado matemático. para la identificación de sus características de trabajo al aplicarlo en la suspensión. El proceso definido se aplicó en función de modelos, variables de diseño, optimización y la tendencia actual en la simulación mecánica. La utilidad del modelo se evaluó para la aplicación de optimización en función del componente objetivo de diseño, considerando varios criterios tales como características cinemáticas, conformidad, estabilidad del vehículo y prueba de comodidad de conducción. Se concluye que el modelo definido sirve como referente para establecer estrategias de simulación y optimización.
\end{abstract}

\section{Review of the Modeling and Optimization Characteristics for the Design of the Macpherson Suspension System}

\begin{abstract}
The objective of this work was to characterize the design processes in the application contexts of simulation software, highlighting the predictive techniques of multibody, finite elements and mathematical modelling for the identification of its work characteristics when applying it in the suspension. The defined process was applied in function of models, design variables, optimization and the current trend in the mechanical simulation. The utility of the model was evaluated for the optimization application in function of the design objective component, considering several criteria such as kinematic characteristics, conformity, vehicle stability and driving ease test. It is concluded that the defined model serves as a reference to establish simulation and optimization strategies.
\end{abstract}




\section{INTRODUCCIÓN}

Uno de los diseños más comunes hoy en día es la suspensión delantera Macpherson, fue descrita por primera vez en una patente de Fiat en 1926, diseñada a finales de los años cuarenta y aplicado en 1948 por Ford en los modelos Anglia y Consul (Sandu et al., 2011). Como tal, es una configuración de suspensión relativamente nueva, por su masiva aplicación en la actualidad (Smith y Swift, 2016). Referidas bajo las normas conocidas como la; SAE J670 e ISO 8855 que abarca los sistemas de coordenadas definidos para el diseño del vehículo/suspensión y el análisis dinámico (J670, 2008; ISO 8855, 2013). Aunque la terminología de dinámica de vehículos definida en el SAE $J 670$ se limita únicamente a los vehículos turismos y camiones ligeros con dos ejes, mientras que la ISO 8855 incluye términos adicionales relativos a vehículos comerciales pesados con ejes múltiples (Cao et al., 2011; Mitra et al., 2015).

El desarrollo de software de simulación virtual ha evolucionado la forma de diseñar nuevos productos que satisfagan las necesidades y requerimientos de los clientes. En esencia, ha permitido el desarrollo de productos virtuales integrados por computadora, completo con modelado 3D integrado, análisis, simulación y optimización, con la mejora continua de las tecnologías de información y comunicación (Horta y Canale, 2009). Además, nacidos del desarrollo de técnicas de ingeniería y diseño computarizado existen muchas opciones predictivas que ofrecen al diseñador y fabricante una mayor confianza en nuevos diseños (Prior, 1992; Colomina et al., 2006). Para el caso de suspensión en vehículos, se requiere una representación precisa de los parámetros de diseño (Hu et al., 2014; Tey et al., 2014) y sobre todo para automatizar el proceso de diseño, se recomienda un software de simulación dinámica combinado con algoritmos de optimización para modelar, simular y sintetizar a fin de lograr resultados precisos (He y McPhee, 2007; Sandu et al., 2011).

La mayoría de las investigaciones sobre el sistema de suspensión se realizan en base al modelo lineal de un cuarto de vehículo, el cual permite una buena representación de la dinámica del sistema para el diseño (Alexandru C. y P. Alexandru, 2011). A fin de obtener mejores resultados, se desarrollan modelos no lineales (mecánico, hidráulico, etc.) y técnicas de simulación multidisciplinaria de sistemas multicuerpo (MBS) (Hurel et al., 2013a). Las técnicas de simulación se aplican ampliamente en todo el desarrollo de la suspensión para optimizar las características cinemáticas $(K)$ y cumplimiento $(C)$ de la suspensión, para predecir el control de frecuencia vertical (Alexandru y Totu, 2016; Aly et al., 2015), el rendimiento de la conducción (R) y manejo (H), y para investigar el rendimiento del viaje (Drotar et al., 2015; Yi et al., 2014). Además, en el proceso de diseño del vehículo la evaluación subjetiva, la prueba objetiva y el análisis de simulación del sistema de estabilidad del vehículo en viaje se aplican al desarrollo de los vehículos (Hurel et al., 2013b; Sun et al., 2013). En este trabajo se aborda los procesos de simulación en los sistemas de suspensión Macpherson, a partir de una revisión de las distintas técnicas aplicadas a la optimización para estos sistemas con el fin de la inclusión de los modelos matemáticos y los avances realizados en el modelado y simulación de la dinámica vehicular.

\section{METODOLOGÍA}

La investigación se realizó en varias etapas: la primera fase se centró en la definición del modelo de aplicación más común del sistema de suspensión, empleando la propuesta metodológica de varios autores como la abordada por Valderrama (2005). Como resultado se identificaron las ecuaciones de movimiento y se establecieron las particularidades de este. Al analizar la literatura encontrada se caracterizó el sistema de optimización a través de las técnicas predictivas de elementos finitos (EF) y de análisis de dinámica en MBS dominantes en la ingeniería computarizada (CAE) de los sistemas de suspensión (Drotar et al., 2015; Liu et al., 2008) con sus modificaciones para la tendencia actual, además de la técnica esquemática por simulink en función del componente objetivo de diseño, asegurándose de que las características $\mathrm{K}$ y $\mathrm{C}$ de la suspensión del vehículo se puedan estimar adecuadamente y también se pueda explicar muchos aspectos de la $\mathrm{R}$ y $\mathrm{H}$ de un vehículo sin problema (Yi et al., 2014). Los sistemas de suspensión tienen que aislar la masa suspendida de la carga propia del vehículo y también proporcionar las características de conducción y manejo deseadas. La mezcla real de cargas introducidas en la suspensión depende en gran medida de la superficie y el uso de la carretera por esa razón es necesario un modelo que aporte a identificar su trabajo de absorción de movimiento (Prior, 1992).

\section{Sistema de suspensión de un cuarto de vehículo}

Las propuestas analíticas para las suspensiones óptimas proporcionan directrices no sólo para la configuración de parámetros, sino más importante, tienen el potencial de ofrecer información sobre el diseño del vehículo. Sancibrian et al., (2010) menciona un mayor rendimiento en el diseño y se logra a medida que aumenta la característica cinemática de la suspensión, dado que la posición del neumático de un vehículo con respecto a la calzada juega un papel importante en la determinación de la manipulación (Sharp y Crolla, 1987). Además, la dependencia de un parámetro óptimo puede modificarse en la fase de concepción del vehículo antes de concebir u optimizar un sistema de suspensión (Smith y Swift, 2016). 
Los sistemas de vehículos y sus parámetros de diseño se examinan para modelos de diferente complejidad. Este modelo es el más simple y se describe todos los fenómenos dinámicos básicos requeridos. La figura 1, se introduce los componentes individuales necesarios para la creación del modelo de vibración, muestra una representación física y consta de una masa suspendida $m_{A}$, una masa no suspendida $m_{B}$, la rigidez de la suspensión $k_{A}$, la rigidez radial del neumático por $k_{B}$, el coeficiente de amortiguamiento viscoso del sistema de suspensión $C_{A}$ y el coeficiente de amortiguamiento viscoso $C_{B}$, además de sus desplazamientos $Z_{A}$ y $Z_{B}$. El símbolo $\zeta(\mathrm{t})$ representa la excitación cinemática dependiente del tiempo de una superficie de la carretera irregular donde el vehículo se desplaza rectilíneamente con una velocidad constante $V$ (Hurel et al., 2013b), todas estas variables están aplicadas en las ecuación 1 y son parte de la matriz de forma mostrada en la ecuación 2, donde se tiene la matriz $M$ como de inercia, $C$ el amortiguamiento viscoso, $K$ la rigidez, $C_{\zeta}$ la excitación transmitida por el amortiguamiento en el neumático, $K_{\zeta}$ la excitación transmitidas por la rigidez radial del neumático, los vectores $q, q^{*}, q^{\prime \prime}$, son los desplazamientos, velocidades y aceleraciones que experimenta el sistema (He y McPhee, 2007).

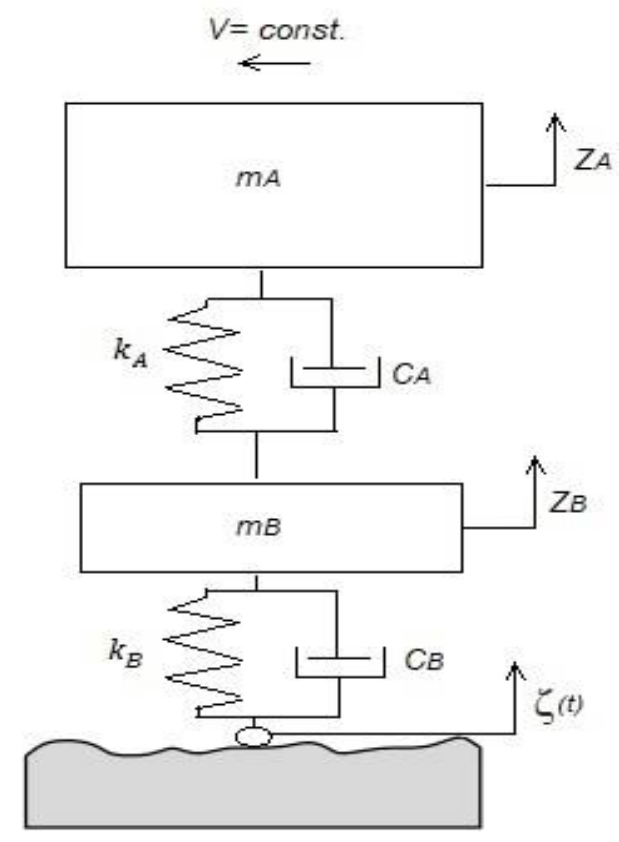

Fig. 1: Modelo de suspensión de un cuarto de vehículo (Adaptada de Lozia y Zdanowicz, 2016; Sandu et al., 2011)

$$
\underbrace{\left[\begin{array}{cc}
m_{A} & 0 \\
0 & m_{B}
\end{array}\right]}_{M} \cdot \underbrace{\left[\begin{array}{l}
\ddot{Z}_{A} \\
\ddot{z}_{B}
\end{array}\right]}_{\dot{q}}+\underbrace{\left[\begin{array}{cc}
c_{1} & -c_{1} \\
-c_{1} & c_{1}+c_{2}
\end{array}\right]}_{C} \cdot \underbrace{\left[\begin{array}{c}
\dot{z}_{A} \\
\dot{z}_{B}
\end{array}\right]}_{\dot{q}}+\underbrace{\left[\begin{array}{cc}
k_{1} & -k_{1} \\
-k_{1} & k_{1}+k_{2}
\end{array}\right]}_{K} \cdot \underbrace{\left[\begin{array}{c}
Z_{A} \\
z_{B}
\end{array}\right]}_{q}=\underbrace{\left[\begin{array}{c}
0 \\
c_{2}
\end{array}\right]}_{c_{\zeta}} \cdot \dot{\zeta}+\underbrace{\left[\begin{array}{c}
0 \\
k_{2}
\end{array}\right] \zeta}_{K_{\zeta}}
$$

$$
M . \ddot{q}+C \cdot \dot{q}+K . q=C_{\zeta} \cdot \dot{\zeta}+K_{\zeta} \cdot \zeta
$$

\section{Sistema de suspensión de un medio vehículo}

En este modelo, el vehículo se representa como una viga con una cierta masa, el haz puede ser asumido como infinitamente rígido, donde se analiza la respuesta dinámica del vehículo sobre las excitaciones de la entrada del terreno $u_{1}$ y $u_{2}$, véase en la figura 2, además está representado por las masas no suspendidas de las ruedas delanteras y traseras $m_{1}, m_{2}$, y la masa de la carrocería del vehículo $m_{3}$. El cuerpo y las ruedas están conectados por suspensiones pasivas con muelles lineales con coeficientes de rigidez $K_{1}, K_{2}$ y amortiguadores viscosos de coeficientes de amortiguación $C_{1}, C_{2}$. Las propiedades elásticas de los neumáticos están representadas por resortes con $k_{t 1}, k_{t 2}$ de rigidez. El modelo longitudinal de la mitad del vehículo es un sistema dinámico con cuatro grados de libertad: desplazamientos verticales de las masas individuales $y_{1}, y_{2}, y_{3}$, rotación lateral de la carrocería del vehículo sobre el centro de gravedad del vehículo también llamado guiño $\varphi_{3}$, relaciona también su inercia $I_{3}$, y los valores de la distancia $b_{1}$ y $b_{2}$ que equilibra el centro de gravedad. 


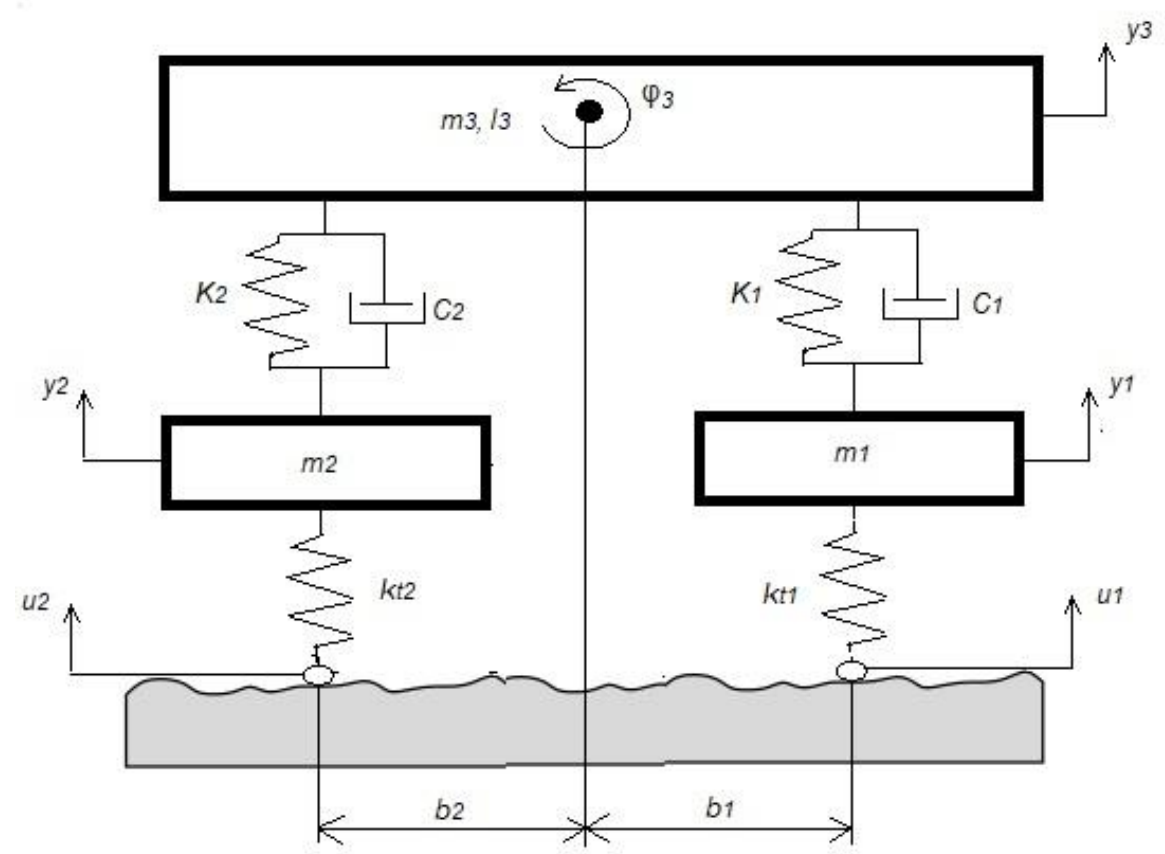

Fig. 2: Modelo de suspensión de medio vehículo (Adaptada de Goga y Klúčik, 2012)

Las ecuaciones $3,4,5,6$ son del movimiento del vehículo para el modelo y se formulan para que se pueda analizar y optimizar su trabajo, respecto a sus operación normal (Goga y Klúčik, 2012).

$$
\begin{aligned}
& m_{1} \ddot{y}_{1}+C_{1}\left(-\dot{y}_{1}+\dot{y}_{3}+b_{1} \dot{\varphi}_{3}\right)+K_{1}\left(-y_{1}+y_{3}+I_{1} \varphi_{3}\right)+k_{t 1}\left(y_{1}-u_{1}\right)=0 \\
& m_{2} \ddot{y_{2}}+C_{2}\left(-\dot{y_{2}}+\dot{y_{3}}-b_{2} \dot{\varphi_{3}}\right)+K_{2}\left(-y_{2}+y_{3}-I_{2} \varphi_{3}\right)+k_{t 2}\left(y_{2}-u_{2}\right)=0 \\
& m_{3} \ddot{y_{3}}+C_{1}\left(-\dot{y_{1}}+\dot{y_{3}}+b_{1} \dot{\varphi_{3}}\right)+C_{2}\left(-\dot{y_{2}}+\dot{y}_{3}-b_{2} \dot{\varphi_{3}}\right)+K_{1}\left(y_{3}-y_{1}+I_{1} \varphi_{3}\right) \\
& +K_{2}\left(-y_{2}+y_{3}-b_{2} \varphi_{3}\right)=0 \\
& I_{3} \ddot{\varphi_{3}}+C_{1} b_{1}\left(-\dot{y_{1}}+\dot{y_{3}}+b_{1} \dot{\varphi}_{3}\right)+C_{2} b_{2}\left(-\dot{y_{2}}+\dot{y}_{3}-b_{2} \dot{\varphi_{3}}\right)+K_{1} b_{1}\left(y_{3}-y_{1}+b_{1} \varphi_{3}\right) \\
& +K_{2} b_{2}\left(-y_{2}+y_{3}-b_{2} \varphi_{3}\right)=0
\end{aligned}
$$

Las herramientas de simulación pueden ser útiles para el análisis cinemático donde se identifican algunas variables que buscan la versatilidad de diseño, un ejemplo, es el uso de un movimiento aplicado en el centro de la rueda para que la acción del movimiento aplicado provoque eliminar los grados de libertad del modelo y, por lo tanto, el análisis es relativamente simple por esta razón es utilizado ampliamente para el diseño y la optimización adecuada, por tal motivo se plantea varios pasos (Liu et al., 2008; Sancibrian et al., 2010) como en la figura 3 para esta revisión.

\section{DISEÑO DEL SISTEMA DE SUSPENSIÓN}

La suspensión crea una conexión entre el vehículo (incluyendo ocupantes y carga) y la carretera. Las fuerzas verticales más notables relaciona el coeficiente de amortiguación, donde los resortes y amortiguadores aporta al vehículo en la reducción de las aceleraciones de la carrocería del vehículo, movimientos reducidos del balanceo y del cabeceo, cambios de la fuerza en la rueda reducidos y comportamiento adecuado de la vibración que es independiente de la carga útil del vehículo, además la energía que se debe controlar aumenta exponencialmente con la velocidad del vehículo (Lee et al., 2009). El diseño ligero del sistema juega un papel más importante en el chasis que en cualquier otra área, ya que toda la masa no suspendida del vehículo se incluye en la suspensión. Una masa no suspendida más pequeña reduce el efecto de las vibraciones de la rueda sobre el cuerpo del vehículo y sobre las fluctuaciones dinámicas de la carga de la rueda (Tey et al., 2014, Rocha-Hoyos et al., 2017). Los componentes presentes en el sistema de suspensión son: carrocería, amortiguador, vástago de pistón, tubo amortiguador, mangueta, barra de dirección, cremallera de dirección, tirante de dirección, brazo inferior, y neumático, estos responden a diferentes grados de libertad (dof); Los dof se calculan utilizando los criterios de Grübler-Kutzbach (Sancibrian et al., 2010). Como vista general se han definido las siguientes restricciones de movimiento visible en la tabla 1. Además, el modelo para el análisis cinemático se presenta en la figura 4 , donde se supone que el chasis está fijo y el centro de la rueda está sujeto a un desplazamiento vertical. 


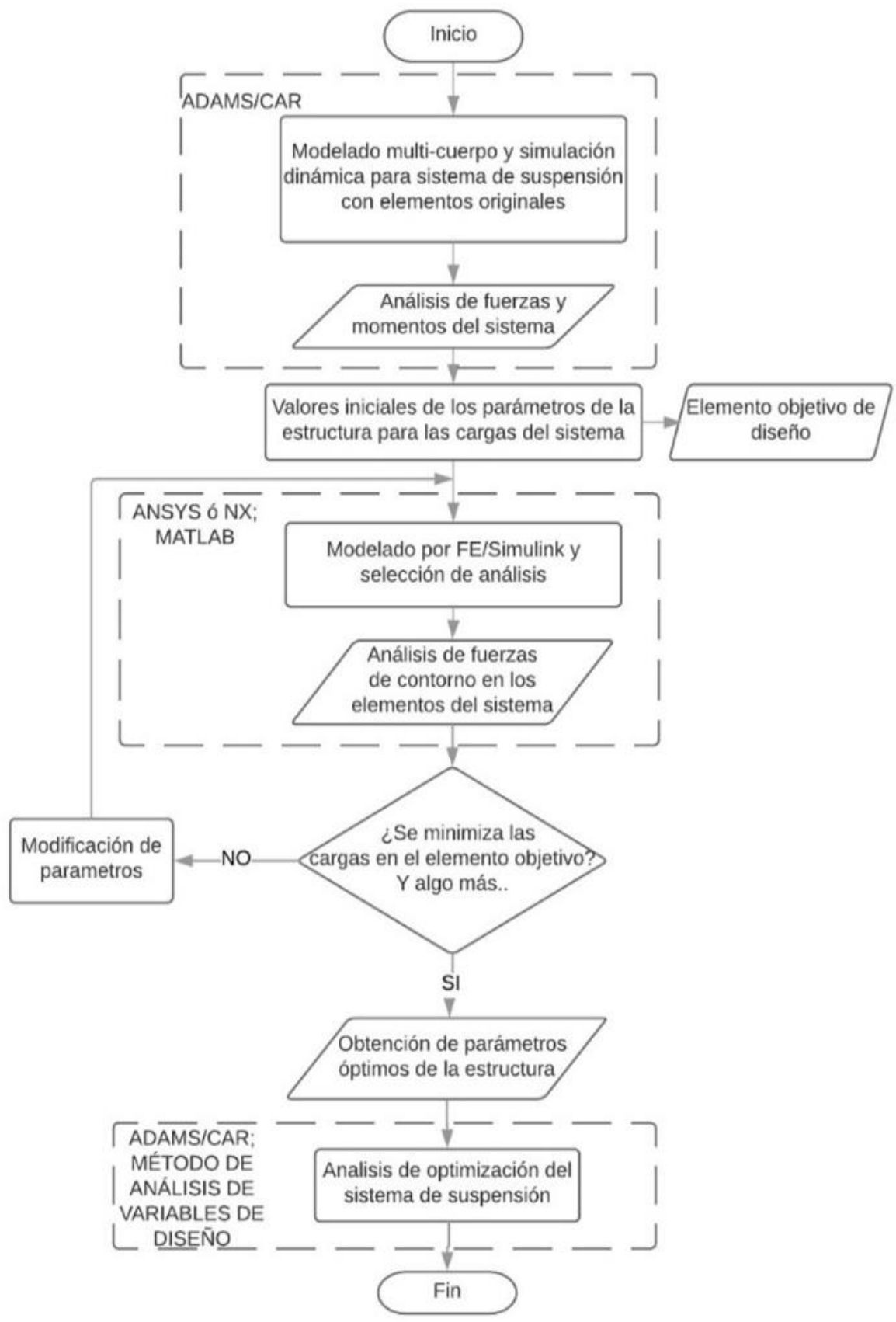

Fig. 3: Secuencia de diseño y optimización de la suspensión (Adaptada de Tey et al., 2014)

Tabla 1: Enlaces y las uniones que conectan los elementos de la suspensión (Adaptada de Hurel et al., 2013b)

\begin{tabular}{|l|c|c|l|}
\hline Unión & dof & Punto de trabajo & Elementos relacionados con las uniones cinemáticas \\
\hline Revolución & 1 & $\mathrm{OF}_{\mathrm{F}}$ & carrocería - brazo inferior \\
\hline Esférico & 3 & $\mathrm{O}_{\mathrm{F}}^{\prime}$ & brazo inferior- amortiguador -mangueta \\
\hline Esférico & 3 & $\mathrm{CF}_{\mathrm{F}}$ & tirante de dirección-amortiguador -mangueta \\
\hline Revolución & 1 & $\mathrm{~T}_{\mathrm{F}}$ & neumático-amortiguador -mangueta \\
\hline Esférico & 3 & $\mathrm{DF}_{\mathrm{F}}$ & barra de dirección - cremallera de dirección \\
\hline Translacional & 1 & $\mathrm{QF}_{\mathrm{F}}$ & cremallera de dirección - carrocería \\
\hline Cilíndrico & 2 & $\mathrm{~B}_{\mathrm{F}}-\mathrm{MF}_{\mathrm{F}}$ & vástago de pistón - tubo amortiguador \\
\hline Esférico & 3 & $\mathrm{~B}_{\mathrm{F}}$ & carrocería- amortiguador \\
\hline
\end{tabular}




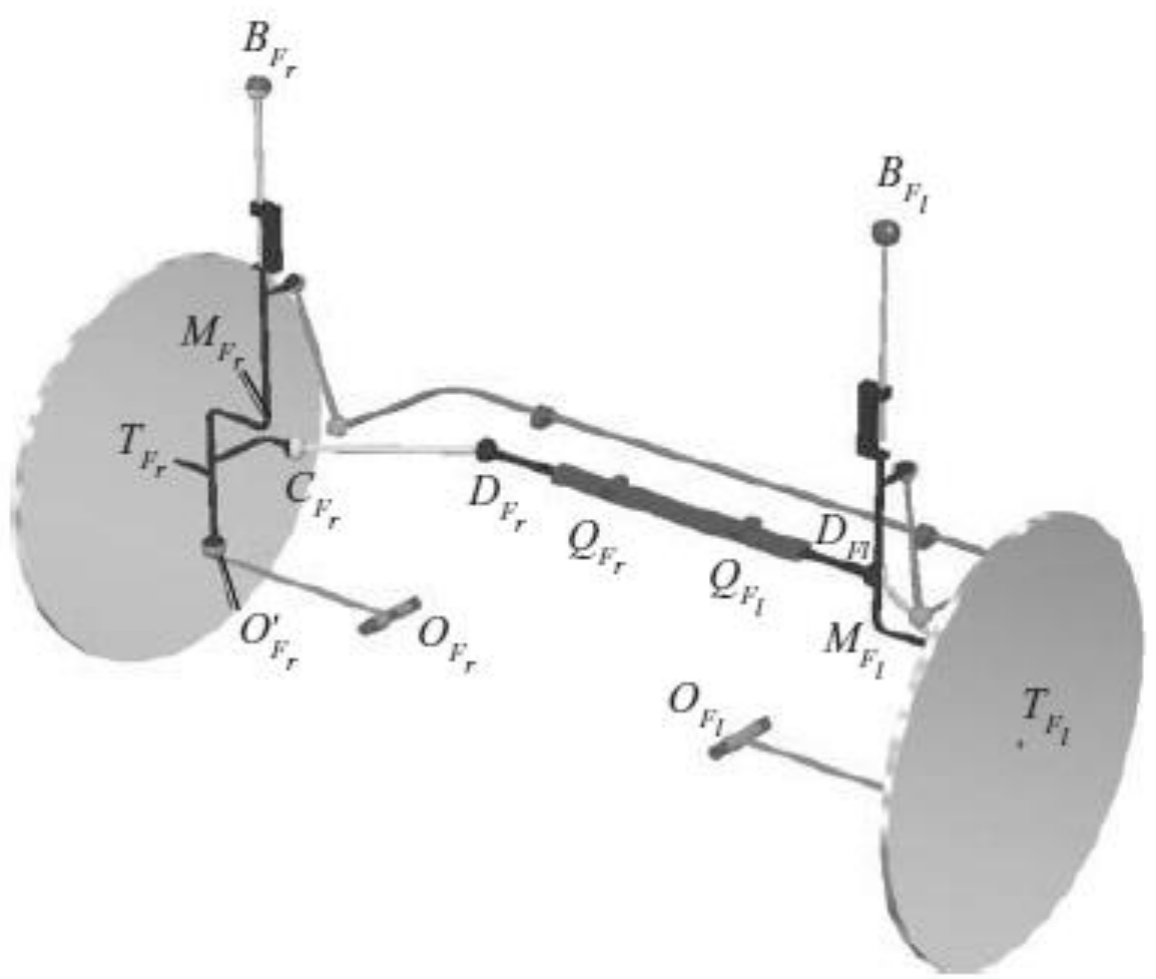

Fig.4: El modelo para el análisis cinemático suspensión (Adaptada de Hurel et al., 2013b; Mántaras y Luque, 2012)

\section{MÉTODOS DE SIMULACIÓN}

Es necesario generalizar las modelizaciones para visualizar el medio de aplicación en función del componente objetivo de diseño.

\section{Estado de frecuencia lineal}

El método lineal da valores propios en el dominio de la frecuencia. Éstos eran muy poderosos al investigar la estabilidad y el comportamiento de conducción para vehículos de pasajeros cuyas suspensiones podían ser lineales de manera fiable. En la actualidad es útil ya que con el mismo se investigan las tendencias y las muestran nociones de su comportamiento de forma generalista (Karnopp, 2009).

\section{Estado estable no lineal}

Para aplicaciones tales como fuerzas en curvas de radio pequeño, transferencia de carga de rueda en una pista curvilínea, etc., son importantes las no linealidades del contacto entre el vehículo y el neumático. Se obtiene una visión valiosa de estos problemas a partir de una solución en estado estacionario, evitando algunas de las complejidades de amortiguación dentro del sistema (Verros et al., 2005)

\section{Dinámica no lineal}

El único enfoque sensible es una solución de integración a tiempo completa de ecuaciones no lineales y este es el método más utilizado en las aproximaciones actuales. Esto permite el modelado de sistemas muy complejos y la obtención de soluciones detalladas. Sin embargo, es posible modelar los componentes con tanto detalle que los datos requeridos para una situación real no pueden obtenerse de manera realista (Mántaras y Luque, 2012).

\section{Bidimensionales y tridimensionales}

Para los análisis de sistemas de suspensión, se deben tener en cuenta los movimientos producidos en los tres ejes, los cuales son considerados por este método, alineándose con los métodos de simulación más recientes (Mántaras y Luque, 2012), los componentes de suspensión son sistemas complejos que deben ser analizados por el método bidimensional y tridimensional (Smith y Swift, 2016). Fallah et al., (2009) propuso un modelo no lineal de un sistema de suspensión macpherson de dos grados de libertad para el control de conducción que se aplica para evaluar los parámetros cinemáticos de la suspensión, tales como ángulos de 
curvas, pivote y bujes principales. Otros modelos bidimensionales se aplican con fines dinámicos, Sandu et al., (2011) desarrollan un modelo dinámico de MBS de una suspensión macpherson para aplicar técnicas de identificación del sistema; Sin embargo, las soluciones exactas requieren el enfoque tridimensional, Sancibrian et al., (2010) propone modelos tridimensionales matemáticos para estudiar la cinemática del sistema macpherson. Mántaras y Luque, (2012) presenta un equipo de prueba virtual con comportamiento elasto-cinemático de un vehículo, en el proceso de diseño, los datos de entrada variable son la aceleración longitudinal, lateral y el radio de la curva, que están definidos por el objeto de diseño.

\section{Modelado simulación dinámica multicuerpo}

Esta técnica se aplica a la simulación de la dinámica y la cinemática de los vehículos, ofreciendo la capacidad de modelar la carga de la carretera y las maniobras de los vehículos con una precisión cada vez mayor, además muestra la optimización de los componentes a un menor costo (Prior, 1992). Hay que considerar que con el software se puede simular numéricamente una gran variedad de matrices de cargas dinámicas reales (Yi et al., 2014). Y como base se muestra el modelo de la suspensión en la figura 5 en Adams/car.

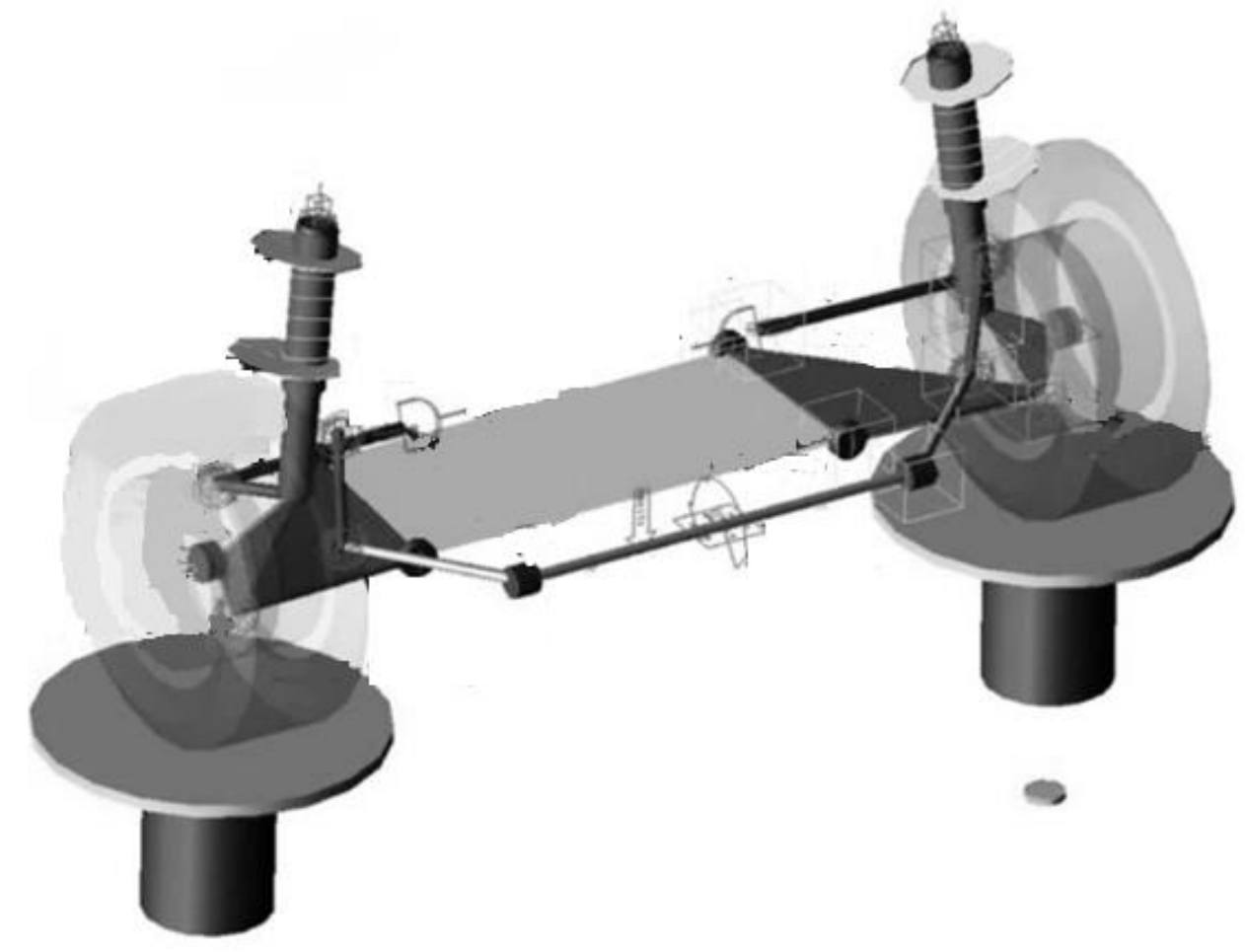

Fig. 5: Modelo de la suspensión delantera en Adams/car (Adaptada de Sun et al., 2013; Tey et al., 2014)

El efecto sobre la orientación de la rueda se puede analizar manipulando cargas cuasi estáticas que representan las fuerzas de frenado y aceleración, y así estimar las cargas severas, además es relevante la maniobra en la conducción. Prior, (1992) investigó la maniobra de cambio de carril que es una de las simulaciones necesarias por tener orientaciones escalonadas o sinusoidales para esto el modelo completo requerido para esta experimentación también requiere que se incluya un modelo de dirección; por otro lado el modelo de simulación del vehículo en movimiento antes del impacto con la calzada muestra que el automotor se encuentra en un movimiento de cabeceo bajo la influencia de la carga de frenado dando como resultado que la dinámica de cualquier punto de referencia para todos los dof se pueda determinar.

Sun et al., (2013) estudiaron el diseño de la suspensión con simulación dinámica MBS y las características K y $\mathrm{C}$, que son fundamentales tanto en teoría como en prueba (Mántaras y Luque, 2012). En este planteamiento según Feng et al., (2012) se identifica los parámetros estructurales que tienen un impacto en las características cinemáticas a través de la simulación de movimiento paralelo de la rueda y optimización de los parámetros, además se mostró que en el proceso de subida y bajada de la rueda, los valores del ángulo de inclinación aumentan ligeramente, los ángulos de dirección y de avance disminuyen ligeramente, lo que significa que la suspensión optimizada mejora la estabilidad de manejo del vehículo y la comodidad de conducción. En otro estudio, Yi et al., (2014) evalúa la suspensión en base a dos etapas para las características cinemáticas $(\mathrm{K})$ y cumplimiento $(\mathrm{C})$, de donde se seleccionan 8 características descritas en la tabla 2 , sabiendo que afectan en gran medida el rendimiento de la conducción $(R)$ y manejo $(H)$, además, 
estas son muy difícil predecirlas con precisión debido a tantas incertidumbres y no linealidades que no se consideran en el modelo de simulación. En el caso particular de no identificar el valor objetivo de cada característica se pueden emplear los resultados de la evaluación de comparación subjetiva y el benchmarking del vehículo de la competencia y el prototipo original del vehículo (Sun et al., 2013; Yi et al., 2014).

Por otra parte, según Drotar et al., (2015) otra utilidad de la técnica, es optimizar las vibraciones táctiles hasta $f=100 \mathrm{~Hz}$ aplicada en la fase inicial del proceso de desarrollo de la suspensión delantera, además para el estudio del confort de conducción, similar a la combinación de las pruebas $\mathrm{K}$ y $\mathrm{C}$ con simulación dinámica MBS para el análisis de dirección y manejo, partiendo del modelo construido en Adams/car. Se demuestra el efecto del aislamiento del bastidor auxiliar sobre el confort de marcha a través del análisis de las fuerzas transmitidas a la carrocería del vehículo, la sensibilidad de la rigidez del buje para impactar la dureza usando un modelo de vehículo completo y además de optimizar la dureza del impacto utilizando diferentes niveles de conformidad entre el bastidor auxiliar y los bujes de suspensión. Liu et al., (2008) estudiaron el sistema de suspensión macpherson con resorte de carga lateral, encontrando las características verticales y laterales deseable sin fuerza longitudinal en operación, lo que reduce notablemente la fuerza lateral y mantiene el rendimiento del sistema original en las simulaciones y experimentos, y puede ser un enfoque eficiente en el diseño óptimo de la estructura, ya que se corrobora también con análisis de elementos finitos. Este enfoque aplica para otros diseños complicados de resortes de suspensión de resorte.

Tabla 2: Características cinemáticas y cumplimiento de la suspensión propuestas (Adaptada de Prior, 1992)

\begin{tabular}{|c|c|}
\hline Parámetro & Características (K y C) \\
\hline rango del cambio de dirección en el desplazamiento de rueda & Cinemáticas \\
\hline cantidad de cambio de dirección en la carrera desplazamiento de rueda & Cinemáticas \\
\hline altura del centro del balanceo & Cinemáticas \\
\hline rango del cambio de dirección del balanceo del vehículo & Cinemáticas y Cumplimiento \\
\hline rigidez lateral de la suspensión & Cumplimiento \\
\hline rango del cambio de dirección en la fuerza lateral & Cumplimiento \\
\hline rigidez longitudinal de la suspensión & Cumplimiento \\
\hline rango del cambio de dirección en la fuerza longitudinal & Cumplimiento \\
\hline
\end{tabular}

Thaller et al., (2016) reportaron estudios utilizando la herramienta de simulación MBS como es Car-Marker y el método de sistema de identificación directa de tiempo continuo (CT Sysld) que sirve para obtener estimaciones precisas de los valores de los parámetros del sistema de suspensión. A través de las pruebas dinámicas que consisten en excitar el sistema de suspensión manejando, también el análisis de uno o varios obstáculos pequeños y registrando la respuesta de este. CT Sysld se utiliza para adaptar los valores de parámetros desconocidos de un modelo de vehículo provisional con el fin de que coincida mejor con los datos medidos. Finamente Sandu et al., (2011) estudió en un auto de la Competencia Grand American para validar los resultados experimentales con los obtenidos en un banco de prueba de macpherson comparado con simulación dinámica MBS donde el modelo no lineal exhibe una coincidencia de aceleración ligeramente mejor que el modelo lineal, analizándose el grado de no linealidad presente en la suspensión.

\section{Modelado elementos finitos}

El análisis de elementos finitos ha sido un enfoque eficiente en el diseño de la estructura para la suspensión de vehículos. Ahorra tiempo y reduce el costo ya que se mantiene el proceso de optimización iterativa en el nivel de los componentes y acá se valida el sistema de suspensión con muelles de carga lateral, y también, para otros diseños complicados de resortes de suspensión de resorte (Liu et al., 2008). Para Lee et al., (1995) la metodología de ingeniería asistida por computadora en fatiga sirve para evaluar cuantitativamente la vida de fatiga de las estructuras automotrices y para identificar áreas críticas y no atenuantes mejorándose el diseño y la reducción de peso, esto es aplicable a los resultados de análisis de elementos finitos elásticos porque el método de densidad de energía de Glinka (enfoque de corrección de plasticidad) se usa en aproximaciones de esfuerzos estáticos y elásticos, además tiene componentes para cumplir con los criterios de durabilidad a través del procedimiento de prueba de vida mecánica y prueba de resistencia real. Bouazara et al., (2016) estudiaron el brazo donde se aplicó el software $A B A Q U S$, como un fuerte software de elementos finitos, al ejecutar el programa para imponer las cargas al brazo inferior donde se detecta la distribución del máximo esfuerzo y el desplazamiento como se muestra en la figura 6 , se observa que las regiones de esfuerzo del brazo inferior que están cerca del lugar de carga impuesta tienen la mayor cantidad de desplazamiento visto en la fig. 6a. 
Generalmente, es obvio que la cantidad de esfuerzo más alta se ha producido en regiones específicas que están cerca del punto fijo en el chasis, como se muestra en la figura $6 \mathrm{~b}$. Al respecto, según Zhang et al., (2015) reportaron que el brazo de la suspensión se analiza en condiciones de carga para garantizar que esté seguro en el proceso de conducción, a través del módulo de análisis de fatiga ANSYS y se verifica que el brazo pueda cumplir con los requisitos de resistencia a la fatiga. Por todo esto la influencia de los componentes de la suspensión sobre las rigideces generales del sistema puede determinarse variando las rigideces de los diversos modelos EF. Según Heißing y Ersoy, (2010) una opción es la optimización topológica de los distintos componentes para alcanzar sus nuevos valores de rigidez, estos nuevos modelos de componentes se cargan en el modelo de simulación Adams/flex, que luego se utiliza para verificar que el sistema global sigue cumpliendo sus objetivos elastocinemáticos, y automatizar el diseño, también se puede reducir espesores de material en aquellas direcciones de carga que no requieren un gran valor de rigidez.

Por otra parte, Li y Song, (2014) establece sus resultados con el nefrograma de esfuerzos para la topología estructural del brazo de suspensión inferior y establece como el punto crítico al extremo de soporte de sujeción el cual tiene un mayor esfuerzo. Entonces, se intensifica parcialmente los materiales en esta área, para evitar el fenómeno de la pérdida de eficiencia. Esto es debido a la abrasión severa en todo tipo de juntas articuladas de suspensión inferior, también se debe plantear un tratamiento de endurecimiento en estos lugares para mejorar su resistencia a la abrasión.

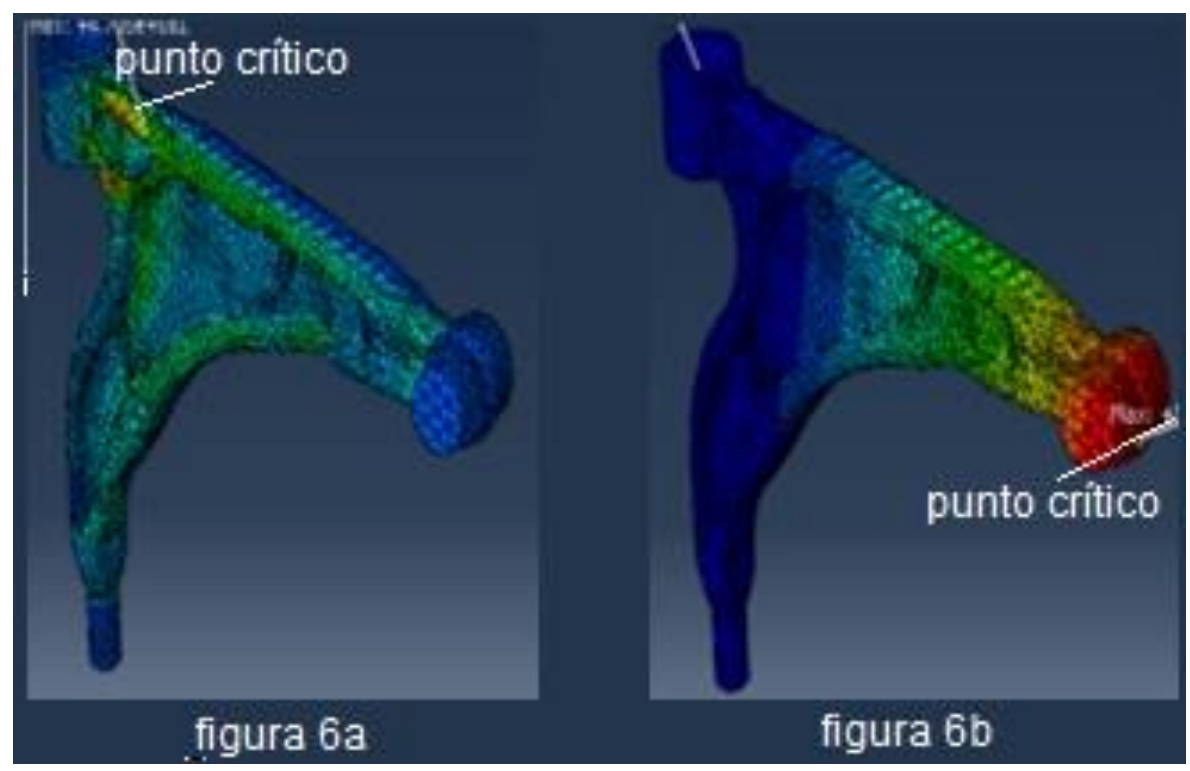

Fig. 6: Brazo inferior de suspensión se visualiza el máximo esfuerzo y el desplazamiento (Adaptada de Bouazara et al., 2016)

\section{Modelado Simulink}

El modelado y la simulación de los sistemas de suspensión resultan fundamental para el análisis de la dinámica vehicular y el diseño de controladores. En este sentido, distintos enfoques han sido propuestos para modelar la interacción con el vehículo, representar las no linealidades de los amortiguadores, o representar la cinemática del sistema de suspensión. Hurel et al., (2013 a) estudiaron la aplicación del modelo de un cuarto de vehículo además se desarrolla ecuaciones diferenciales, la función de transferencia, y ecuaciones de estado para las soluciones y simulaciones en simulink y simMechanics. Goga y Klúčik, (2012) estudiaron los algoritmos genéticos en los parámetros de suspensión pasiva con Matlab/Simulink en el modelo matemático semioruga donde se presenta resultados del modelo optimizados, esto muestra una disminución significativa de las amplitudes y una estabilización más rápida de las cantidades medidas frente a los resultados del modelo con los parámetros originales. Los criterios objetivos fueron analizar la comodidad del pasajero y la estabilidad de conducción.

Hurel et al., (2013 b) propone incluir el ángulo de inclinación y el ancho de la vía, que no pueden abordarse con el modelo convencional de cuarto de auto, a partir del modelo cinemático-dinámico propuesto que se ha comparado con un modelo realista de Adams/view para analizar el comportamiento asimétrico no lineal. En este caso no solo se considera el movimiento vertical de la masa suspendida sino también: rotación y traslación de la masa no suspendida; masa de la rueda y su momento de inercia alrededor del eje longitudinal; 
amortiguación del neumático y deflexión lateral debido al mecanismo de suspensión. Estas mejoras a partir del proceso sistemático del modelo planar, así como el conjunto completo de ecuaciones matemáticas para la implementación reproducible de simulink se obtiene nuevos resultados experimentales utilizando diferentes puntos clave de suspensión y parámetros dinámicos (Hurel et al., 2013 b).

Ahora bien, Mántaras y Luque, (2012) desarrollaron un equipo de prueba que permite la optimización cinemática de la suspensión, una vez que el vehículo ha sido fabricado como es el caso de los vehículos de competición, la medición de diferentes sensores en tiempo real aportan para demostrar la validez del modelo tridimensional de un vehículo con una suspensión de dirección tipo macpherson delantera y suspensión posterior para evaluar el comportamiento dinámico o la comodidad del vehículo completo, y en esencia la ventaja de diseño es la posibilidad de conocer la posición relativa de los diferentes componentes del vehículo respecto del suelo, se mejoró ya que los resultados de simulación de Adams/car se usaran como entradas en el modelo matlab, se calcula y contrasta.

En la figura 7 se encuentra el modelo del vehículo donde requiere una serie de datos geométricos, coordenadas de las estructura del vehículo como las dimensiones de los componentes del sistema de suspensión y dirección; además la aceleración longitudinal $a_{x}$, lateral $a_{y}$ y el radio de la curva, todas en función del tiempo para permitir establecer el sistema de ecuaciones del vehículo cinemático. Como parámetros dinámicos complementarios son la masa del vehículo, la distribución de masa (delantera-trasera), la relación de transmisión, la relación de frenado y el coeficiente de rigidez vertical de las suspensiones (delantera y trasera). Y por lo tanto visualizar el ángulo de balanceo $\theta$, ángulo de cabeceo $\varphi$, el ángulo de convergencia $\delta$ y la altura del centro de gravedad $z_{c d g}$ todo esto en relación al comportamiento de la suspensión.

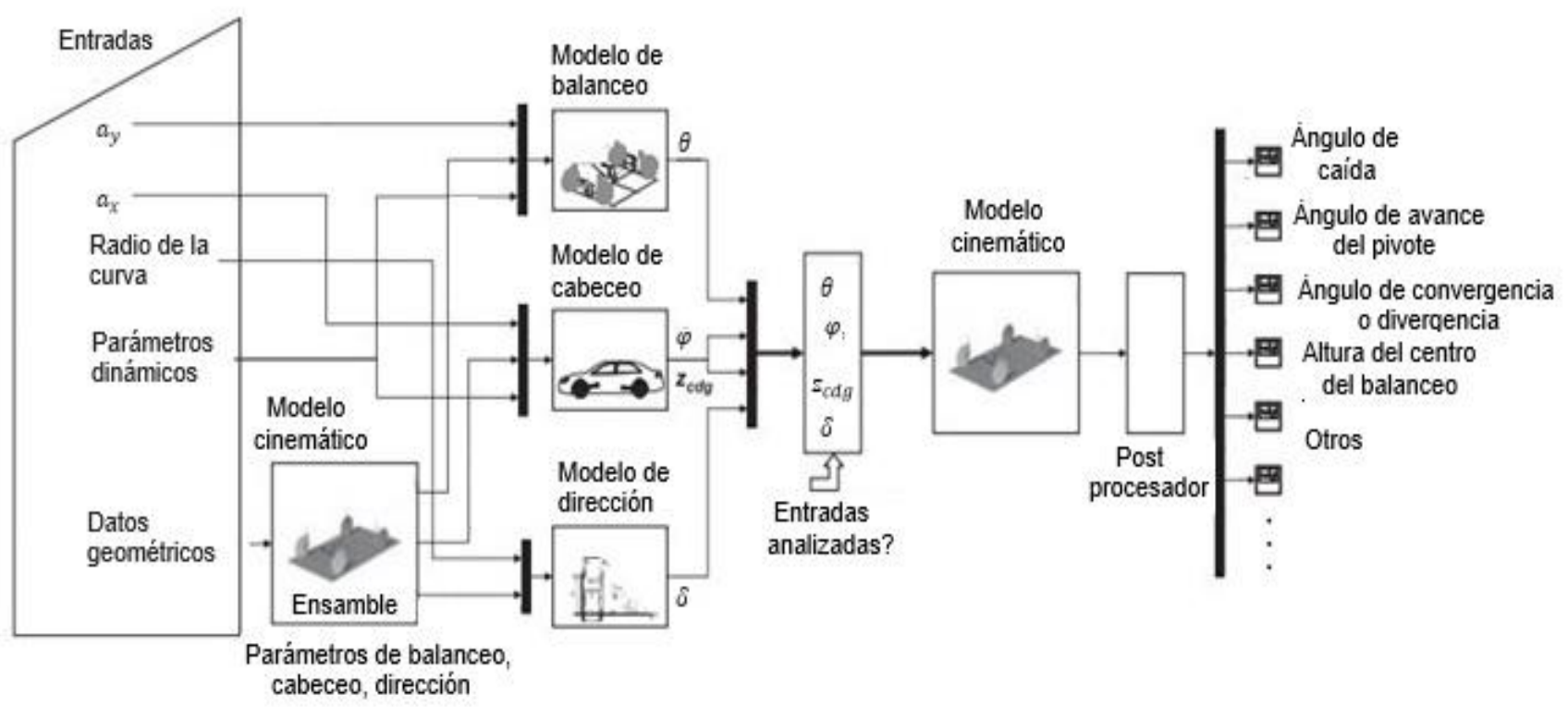

Fig. 7: Equipo de pruebas en Matlab/Simulink (Adaptada de Mántaras y Luque, 2012; Sun et al., 2013)

\section{ANÁLISIS DE OPTIMIZACIÓN}

La optimización del sistema está enfocada en reducir costos respecto a pruebas experimentales, esto es uno de los aspectos de estudio que concierne al desarrollo. Para esto según Sun et al., (2013) analizar los datos de prueba del sistema de suspensión por simulación MBS mediante los criterios K y C, la estabilidad de la operación del vehículo y la prueba de confort de conducción es adecuado por el análisis con CarSim para confirmar el rendimiento del sistema y del vehículo. Por otro lado, Tey et al., (2014) investigaron un enfoque eficiente para identificar los parámetros desconocidos a través de la optimización basada en resultados experimentales a partir del modelo construido en Adams/Car, luego se utiliza la estrategia de adaptaciónevolución de la matriz de covarianza (CMA-es) combinada con un pseudo-objetivo en los parámetros de diseño para mejorar la simulación, reduciendo el número de objetivos de 40 a 30 y dando como resultado que los parámetros de diseño son capaces de predecirse con precisión como las características $\mathrm{K}$ y $\mathrm{C}$ de la suspensión física. En el caso de utilizar la técnica de suavidad de conducción basada en CAE, las decisiones arquitectónicas de chasis impulsadas por datos se pueden realizar mucho antes de los prototipos físicos (Drotar et al., 2015). 
Uno de los temas que ha guiado la optimización ha sido el análisis de sensibilidad. Lee et al. (2009) estudiaron el factor de diseño estático cinemático en cada articulación de la suspensión, a partir de esto, se predice las variaciones del ángulo de caída, ángulo de avance del pivote, ángulo de convergencia y divergencia y la optimización en la suspensión tipo macpherson respecto a las fuerzas de reacción en las uniones, a partir de los cuales se pueden encontrar los efectos de cada punto de trabajo (variable de diseño) necesarios para las características del vehículo en las primeras etapas de diseño. En una etapa complementaria a esto, al aplicar una herramienta estadística para la mejora de la calidad en el producto, según Feng et al., (2012), se aplica diseño de experimento (DoE) al análisis de sensibilidad de la cinemática de suspensión. Yi et al., (2014) menciona la oportunidad de mejora integral a través de la utilización del enfoque de diseño axiomático (AD) donde el proceso de optimización es mucho más rápido y más estable, para las características de suspensión de $\mathrm{K}$ y $\mathrm{C}$, ya que se desarrolla una función multiobjetivo que se define para un proceso de optimización efectivo, y luego se aplica un algoritmo basado en gradiente a cada problema. En otro estudio Mitra et al., (2015) plantea que para garantizar la comodidad de conducción óptima según las normas ISO 2631-1: 1997 se complementa utilizando DoE y así encontrar la configuración óptima en los parámetros como la presión de los neumáticos, el coeficiente de amortiguación, la rigidez, la masa de los muelles, la inclinación, la convergencia y la velocidad de las ruedas.

Una perspectiva es la optimización por el análisis multicriterio de los mecanismos de suspensión donde Alexandru y Totu, (2016) considera el objetivo de minimizar tanto como sea posible en términos de requisitos funcionales y constructivos los siguientes: ángulo de avance del pivote, la distancia entre ejes, el ángulo de convergencia o divergencia y las variaciones del ángulo de inclinación de las ruedas. El proceso de optimización puede ampliarse al considerar más objetivos que intervienen en el sistema de suspensión. Steffen et al., (2015) lograron describir un método de aplicación de vehículo completo con MBS (> 100 dof) en tiempo real, esto permite evaluar de manera eficiente el rendimiento de manejo de todo el vehículo. Se puede evaluar una matriz de parámetros de diseño del chasis (geometrías, cinemáticas) en combinación con sistemas de suspensión, dirección y tren motriz, además se pueden lograr las mejores combinaciones utilizando métodos DoE en su optimización. Por ende, el campo de estudio en esta área sigue siendo amplio para desarrollar investigaciones.

\section{CONCLUSIONES}

De acuerdo a los resultados de este estudio, y su análisis se pueden extraer las siguientes conclusiones principales:

1) La modelación de la suspensión pasiva converge hacia la búsqueda de soluciones alternativas en la optimización de las características cinemáticas $(K)$ y cumplimiento $(C)$ de la suspensión del vehículo, además de la estimación adecuada del rendimiento de la conducción $(R)$ y manejo $(H)$;

2) Los avances recientes en la dinámica y el diseño de la suspensión de vehículos, han contribuido considerablemente a las mejoras del rendimiento y la seguridad dinámica, así como a la facilidad de conducción;

3) El modelo matemático de un cuarto y de un medio de vehículo aplicado a la suspensión Macpherson ha demostrado ser un modelo flexible aplicable a cualquier configuración, con él es posible calcular la variación en la geometría de la suspensión.

\section{REFERENCIAS}

Alexandru, C. y P. Alexandru, A Comparative Analysis between the Vehicles Passive and Active Suspensions, Int. J. Mech., 4(5), 371-378 (2011)

Alexandru, C. y V. Totu, Method for the Multi-Criteria Optimization of Car Wheel Suspension Mechanisms, doi: 10.15446/ing.investig.v36n2.52517, Ing. Investig., 36(2), 60-67 (2016)

Aly, M. F., A. O. Nassef y K. Hamza, Multi-Objective Design of Vehicle Suspension Systems Via a Local Diffusion Genetic Algorithm for Disjoint Pareto Frontiers, doi: 10.1080/0305215X.2014.918113, Eng. Opt., 47(5), 706-717 (2015)

Bouazara, M., H. Banitalebi, K. A. Ragab y H. Mrad, On the Characteristics of Automotive Low Arm-Suspension System Parts Made of Aluminum Casting Alloys, doi: 10.1080/13640461.2015.1106782, Inter. J. Cast Metal Res., 29(3), 129$136(2016)$

Cao, D., X. Song y M. Ahmadian, Editors' Perspectives: Road Vehicle Suspension Design, Dynamics, and Control, doi: 10.1080/00423114.2010.532223, Veh. Syst. Dyn., 49(1-2), 3-28 (2011)

Colomina, F.J., J. Masiá, T.V. Esquerdo y J. F. Dols, Modelado Estructural de Componentes de Bastidores de Vehículos Pesados mediante el Método de Elementos Finitos, doi:10.4067/S0718-07642006000600006, Inf. Tecnol., 17(6), 2734 (2006) 
Drotar, T., J. Palandri, F. Wolf-Monheim, P. Zandbergen y B. Reff, CAE-Based Driving Comfort Optimization of Passenger Cars, doi: 10.4271/2015-01-1583, SAE Intern. J. of Pass. Cars-Mech. Sys. 8(2015-01-1583), 703-710 (2015)

Fallah, M.S., R. Bhat y W.F. Xie, New Model and Simulation of Macpherson Suspension System for Ride Control Applications, Veh. Syst. Dyn., 47(2), 195-220 (2009)

Feng, J.Z., J.T. Song y W.H. Zhu, Optimization Design of Vehicle Front Suspension Structure Parameters Using DOE Method, App. Mech. and Mat. Trans Tech Pub., 120, 375-380 (2012)

Goga, V. y M. Klúčik, Optimization of Vehicle Suspension Parameters with Use of Evolutionary Computation, Proc. Eng., 48, 174-179 (2012)

He, Y. y J. McPhee, Application of Optimisation Algorithms and Multibody Dynamics to Ground Vehicle Suspension Design, Int. J. Heavy Veh. Syst., 14(2), 158-192 (2007)

Heißing, B. y M. Ersoy, Chassis Handbook: Fundamentals, Driving Dynamics, Components, Mechatronics, Perspectives, $1^{\text {a }}$ Ed., 70-86, Springer Science and Business Media, Berlin, Alemania (2010)

Horta, J. y A. Canale, Influencia de los Parámetros de Carga en la Estabilidad Direccional de un Vehículo Combinado, doi: 10.4067/S0718-07642009000400015, Inf. Tecnol., 20(4), 131-136 (2009)

Hu, Y., M. Z. Chen y Z. Shu, Passive Vehicle Suspensions Employing Inerters With Multiple Performance Requirements, J. Sound Vibr., 333(8), 2212-2225 (2014)

Hurel, J., A. Mandow y A. Cerezo, Los Sistemas de Suspensión Activa y Semiactiva: una Revisión, Rev. Iberoa. Autom. Inf. Ind. RIAI, 10(2), 121-132 (2013a)

Hurel, J., A. Mandow y A. García-Cerezo, Kinematic and Dynamic Analysis of the McPherson Suspension with a Planar Quarter-Car Model, doi: 10.1080/00423114.2013.804937, Veh. Syst. Dyn., 51(9), 1422-1437 (2013b)

ISO, D. 8855, Road Vehicles - Vehicle Dynamics and Road-Holding Ability-Vocabulary. DIN Deutsches Institut Für Normung e, (2013)

J670, S.S., Vehicle Dynamics Terminology, Vehicle Dynamics Standards Committee, Warrendale, PA, USA, SAE (2008)

Karnopp, D., How Significant are Transfer Function Relations and Invariant Points for a Quarter car Suspension Model? Veh. Syst. Dyn., 47(4), 457-464 (2009)

Lee, H.G., C. J. Won y J. W. Kim, Design Sensitivity Analysis and Optimization of McPherson Suspension Systems, Proc. World Congress Eng., 2, 1-3 (2009)

Lee, Y. L., M. N. Raymond y M. A. Villaire, Durability Design Process of a Vehicle Suspension Component, J. Testing Eval., 23(5), 354-363 (1995)

Li, L. Z. y Z. F. Song, Finite Element Simulation of Front Suspension Lower Arm of MacPherson Type Based on ANSYS, Appl. Mech. Mat. Trans Tech Publi., 577, 162-165 (2014)

Liu, J., D. J. Zhuang, F. Yu y L. M. Lou, Optimized Design for a MacPherson Strut Suspension with Side Load Springs, Inter. J. Aut. Tech., 9(1), 29-35 (2008)

Lozia, Z. y P. Zdanowicz, Optimization of Damping in the Passive Automotive Suspension System With Using Two QuarterCar Models, IOP Conf. Ser.: Mat. Sci. y Eng., 148, 012014 (2016)

Mántaras, D. A. y P. Luque, Virtual Test Rig to Improve The Design and Optimisation Process of the Vehicle Steering and Suspension Systems, Veh. Syst. Dyn., 50(10), 1563-1584 (2012)

Mitra, A.C., T. Soni, G.R. Kiranchand, S. Khan y N. Banerjee, Experimental Design and Optimization of Vehicle Suspension System, Mat. Today: Proc., 2(4-5), 2453-2462 (2015)

Prior, G. M., The Use of Multi-Body Systems Analysis in the Design and Analysis of Vehicle Suspension Systems, SAE Tech. Paper No. 921463 (1992)

Rocha-Hoyos, J., L. E. Tipanluisa, S. W. Reina y C. R. Ayabaca, Evaluación del Sistema de Tracción en un Vehículo Eléctrico Biplaza de Estructura Tubular, doi: 10.4067/S0718-07642017000200004, Inf. Tecnol., 28(2), 29-36 (2017)

Sancibrian, R., P. Garcia, F. Viadero, A. Fernandez y A. De-Juan, Kinematic Design of Double-Wishbone Suspension Systems Using a Multiobjective Optimisation Approach, Veh. Sys. Dyn., 48(7), 793-813 (2010)

Sandu, C., E. R. Andersen y S. Southward, Multibody Dynamics Modelling and System Identification of a Quarter-Car Test Rig With McPherson Strut Suspension, Veh. Syst. Dyn., 49(1-2), 153-179 (2011)

Sharp, R. S. y D. A. Crolla, Road Vehicle Suspension System Design-a Review, Veh. Syst. Dyn., 16(3), 167-192 (1987)

Smith, M. C. y S. J. Swift, Design of Passive Vehicle Suspensions for Maximal Least Damping Ratio, Veh. Syst. Dyn., 54(5), 568-584 (2016)

Steffen, S., S. Eberhard, S. Waldemar y H. Josef, Optimization of Vehicle Handling Performance Using a Full Vehicle Model With Multi-Body System (MBS) Suspensions in Multiple Real Time-Applying the DoE Method, Inter. J. Automotive Eng., 6(2), 53-57 (2015) 
Sun, F., J. Jiang, W. Liu, Z. Pan y F. Zhao, Optimization Design of Suspension Structure Based on Multi-Body Dynamic Analysis, Proc. FISITA 2012 World Aut. Cong.-Springer, Berlin, Heidelberg, 43-56 (2013)

Tey, J.Y., R. Ramli, C.W. Kheng, S. Y. Chong y M. A. Z. Abidin, Identification of Vehicle Suspension Parameters by Design Optimization, Eng. Optim., 46(5), 669-686 (2014)

Thaller, S., F. Reiterer, R. Schmied, H. Waschl, H. Kokal y L. del Re, Fast Determination of Vehicle Suspension Parameters Via Continuous Time System Identification, IFAC, 49(11), 448-453 (2016)

Valderrama, J. O., Principales Aspectos sobre la Preparación de un Artículo para ser Publicado en una Revista Internacional de Corriente Principal, doi: 10.4067/S0718-07642005000200002, Inf. Tecnol., 16(2), 3-14 (2005)

Verros, G., S. Natsiavas y C. Papadimitriou, Design Optimization of Quarter-Car Models With Passive and Semi-Active Suspensions under Random Road Excitation, Modal Analysis, 11(5), 581-606 (2005)

Yi, Y.S., J. Park y K. J. Hong, Design Optimization of Suspension Kinematic and Compliance Characteristics, doi: 10.4271/2014-01-0394, SAE Tech. Paper No. 2014-01-0394 (2014)

Zhang, H.B., R.J. Zhang e Y. Chang, Finite Element Analysis of Automobile Suspension Control Arm, doi: 10.4028/www.scientific.net/AMM.752-753.859, Appl. Mech. Mat. Trans Tech. Publ., 752, 859-863 (2015) 
